\title{
EXACT CONTROLLABILITY OF A LINEAR KORTEWEG-DE VRIES EQUATION BY THE FLATNESS APPROACH
}

\author{
PHILIPPE MARTIN, IVONNE RIVAS, LIONEL ROSIER, AND PIERRE ROUCHON
}

\begin{abstract}
We consider a linear Korteweg-de Vries equation on a bounded domain with a left Dirichlet boundary control. The controllability to the trajectories of such a system was proved in the last decade by using Carleman estimates. Here, we go a step further by establishing the exact controllability in a space of analytic functions with the aid of the flatness approach.
\end{abstract}

\section{Mathematics Subject Classification: 37L50, 93B05}

Keywords: Korteweg-de Vries equation; exact controllability; controllability to the trajectories; flatness approach; Gevrey class; smoothing effect.

\section{INTRODUCTION}

The Korteweg-de Vries (KdV) equation is a well-known dispersive equation that may serve as a model for the propagation of gravity waves on the surface of a canal or a lake. It reads

$$
\partial_{t} y+\partial_{x}^{3} y+y \partial_{x} y+\partial_{x} y=0,
$$

where $t$ is time, $x$ is the horizontal spatial coordinate, and $y=y(x, t)$ stands for the deviation of the fluid surface from rest position. As usual, $\partial_{t} y=\partial y / \partial t, \partial_{x} y=\partial y / \partial x, \partial_{x}^{3} y=\partial^{3} y / \partial x^{3}$, etc.

When the equation is considered on a bounded interval $(0, L)$, it has to be supplemented with three boundary conditions, for instance

$$
y(0, t)=u(t), \quad y(L, t)=v(t), \quad \partial_{x} y(L, t)=w(t),
$$

and an initial condition

$$
y(x, 0)=y_{0}(x) .
$$

The controllability of the Korteweg-de Vries equation with various boundary controls has been considered by many authors since several decades (see e.g. the surveys [18, 2]). The exact controllability in the energy space $L^{2}(0, L)$ was derived by Rosier in [15] (resp. by Glass and Guerrero in [6]) with $w$ as the only control input (resp. with $v$ as the only control input). On the other hand, if we take $u$ as the only control input, the exact controllability fails in the energy space [17], because of the smoothing effect. Nevertheless, both the null-controllability and the controllability to the trajectories hold with the left Dirichlet boundary control, see [17] and [5]. The aim of the present paper is to go a step further by investigating the exact controllability in a "narrow" space with the left Dirichlet boundary control. Due to the smoothing effect, the space in which the exact controllability can hold is a space of analytic functions. For the sake of simplicity, we will focus on a linear $\mathrm{KdV}$ equation (removing the nonlinear term $y \partial_{x} y$ ). Performing a scaling in time and space, there is no loss of generality in assuming that $L=1$. 
By a translation, we can also assume that $x \in(-1,0)$. The first-order derivative term will assume the form $a \partial_{x} y$ where $a \in \mathbb{R}_{+}$is some constant. The case $a=1$ corresponds to the linearized KdV equation

$$
\partial_{t} y+\partial_{x}^{3} y+\partial_{x} y=0
$$

while the case $a=0$ corresponds to the "simplified" linearized KdV equation

$$
\partial_{t} y+\partial_{x}^{3} y=0
$$

which is often considered when investigating the Cauchy problem on the line $\mathbb{R}$ (instead of a bounded interval) by doing the change of unknown $\tilde{y}(x, t)=y(x+t, t)$.

The paper will be concerned with the control properties of the system:

$$
\begin{aligned}
\partial_{t} y+\partial_{x}^{3} y+a \partial_{x} y=0, & x \in(-1,0), t \in(0, T), \\
y(0, t)=\partial_{x} y(0, t)=0, & t \in(0, T), \\
y(-1, t)=u(t), & t \in(0, T), \\
y(x, 0)=y_{0}(x), & x \in(-1,0),
\end{aligned}
$$

where $y_{0}=y_{0}(x)$ is the initial data and $u=u(t)$ is the control input.

We shall address the following issues:

1. (Null controllability) Given any $y_{0} \in L^{2}(-1,0)$, can we find a control $u$ such that the solution $y$ of (1.6)-(1.9) satisfies $y(., T)=0$ ?

2. (Reachable states) Given any $y_{1} \in \mathcal{R}$ (a subspace of $L^{2}(-1,0)$ defined thereafter), can we find a control $u$ such that the solution $y$ of (1.6)-(1.9) with $y_{0}=0$ satisfies $y(., T)=y_{1}$ ?

We shall investigate both issues by the flatness approach and derive an exact controllability in $\mathcal{R}$ by combining our results.

The null controllability of (1.6)-(1.9) was established in [17] (see also [5]) by using a Carleman estimate. The control input $u$ was found in a Sobolev space (e.g. $u \in H^{\frac{1}{2}-\varepsilon}(0, T)$ for all $\varepsilon>0$ if $y_{0} \in L^{2}(-1,0)$, see [5]). Here, we shall improve this result by designing a control input in a Gevrey class. Furthermore, the trajectory and the control will be given explicitly as the sums of series parameterized by the flat output. To state our result, we need introduce a few notations. A function $u \in C^{\infty}\left(\left[t_{1}, t_{2}\right]\right)$ is said to be Gevrey of order $s \geq 0$ on $\left[t_{1}, t_{2}\right]$ if there exist some constant $C, R \geq 0$ such that

$$
\left|\partial_{t}^{n} u(t)\right| \leq C \frac{(n !)^{s}}{R^{n}} \quad \forall n \in \mathbb{N}, \forall t \in\left[t_{1}, t_{2}\right] .
$$

The set of functions Gevrey of order $s$ on $\left[t_{1}, t_{2}\right]$ is denoted by $G^{s}\left(\left[t_{1}, t_{2}\right]\right)$. A function $y \in C^{\infty}\left(\left[x_{1}, x_{2}\right] \times\right.$ $\left.\left[t_{1}, t_{2}\right]\right)$ is said to be Gevrey of order $s_{1}$ in $x$ and $s_{2}$ in $t$ on $\left[x_{1}, x_{2}\right] \times\left[t_{1}, t_{2}\right]$ if there exist some constants $C, R_{1}, R_{2}>0$ such that

$$
\left|\partial_{x}^{n_{1}} \partial_{t}^{n_{2}} y(x, t)\right| \leq C \frac{\left(n_{1} !\right)^{s_{1}}\left(n_{2} !\right)^{s_{2}}}{R_{1}^{n_{1}} R_{2}^{n_{2}}} \quad \forall n_{1}, n_{2} \in \mathbb{N}, \forall(x, t) \in\left[x_{1}, x_{2}\right] \times\left[t_{1}, t_{2}\right] .
$$

The set of functions Gevrey of order $s_{1}$ in $x$ and $s_{2}$ in $t$ on $\left[x_{1}, x_{2}\right] \times\left[t_{1}, t_{2}\right]$ is denoted by $G^{s_{1}, s_{2}}\left(\left[x_{1}, x_{2}\right] \times\right.$ $\left.\left[t_{1}, t_{2}\right]\right)$.

The first main result in this paper is a null controllability result with a control input in a Gevrey class.

Theorem 1.1. Let $y_{0} \in L^{2}(-1,0), T>0$, and $s \in\left[\frac{3}{2}, 3\right)$. Then there exists a control input $u \in G^{S}([0, T])$ such that the solution y of (1.6)-(1.9) satisfies $y(., T)=0$. Furthermore, it holds that

$$
y \in C\left([0, T], L^{2}(-1,0)\right) \cap G^{\frac{s}{3}, s}([-1,0] \times[\varepsilon, T]) \quad \forall \varepsilon \in(0, T) .
$$


The second issue investigated in this paper is the problem of the reachable states. For the heat equation, an important step in the characterization of the reachable states was given in [11] with the aid of the flatness approach. It was proven there that reachable states can be extended as holomorphic functions on some square of the complex plane, and conversely that holomorphic functions defined on a ball centered at the origin and with a sufficiently large radius give by restriction to the real line reachable states. See also [4] for an improvement of this result as far as the domain of analyticity of the reachable states is concerned.

To the best knowledge of the authors, the determination of the reachable states for (1.6)-(1.9) has not been addressed so far. From the controllability to the trajectories established in [17, 5], we know only that any function $y_{1}=y_{1}(x)$ that can be written as $y_{1}(x)=\bar{y}(x, T)$ for some trajectory $\bar{y}$ of (1.6)-(1.9) associated with some $y_{0} \in L^{2}(-1,0)$ and $u=0$ is reachable. But such a function is in $G^{\frac{1}{2}}([-1,0])^{1}$ with $y_{1}(-1)=0$ and $\left(\partial_{x}^{3}+a \partial_{x}\right)^{n} y_{1}(-1)=0$ for all $n \geq 1$, according to Proposition 2.1 (see below). Proceeding as in [11], we shall obtain a class of reachable states that are less regular than those for the controllability to the trajectories (namely $y_{1} \in G^{1}([-1,0]$ ) for which no boundary condition has to be imposed at $x=-1$.

To state our second main result, we need to introduce again some notations. For $z_{0} \in \mathbb{C}$ and $R>0$, we denote by $D\left(z_{0}, R\right)$ the open disk

$$
D\left(z_{0}, R\right):=\left\{z \in \mathbb{C} ;\left|z-z_{0}\right|<R\right\}
$$

and by $H\left(D\left(z_{0}, R\right)\right)$ the set of holomorphic (i.e. complex analytic) functions on $D\left(z_{0}, R\right)$. Introduce the operator

so that (1.6) can be written

$$
P y:=\partial_{x}^{3} y+a \partial_{x} y,
$$

$$
\partial_{t} y+P y=0 .
$$

Since $\partial_{t}$ and $P$ commute, it follows from (1.11) that for all $n \in \mathbb{N}^{*}$

$$
\partial_{t}^{n} y+(-1)^{n-1} P^{n} y=0
$$

where $P^{n}=P \circ P^{n-1}$ and $P^{0}=I d$. We are in a position to define the set of reachable states: for any $R>1$, let

$$
\mathcal{R}_{R}:=\left\{y \in C^{0}([-1,0]) ; \exists z \in H(D(0, R)), y=z_{\mid[-1,0]} \text {, and }\left(P^{n} y\right)(0)=\partial_{x}\left(P^{n} y\right)(0)=0 \quad \forall n \geq 0\right\} .
$$

The following result is the second main result in this paper.

Theorem 1.2. Let $a \in \mathbb{R}_{+}, T>0$, and $R>R_{0}:=e^{(3 e)^{-1}}(1+a)^{\frac{1}{3}}>1$. Pick any $y_{1} \in \mathcal{R}_{R}$. Then there exists a control input $u \in G^{3}([0, T])$ such that the solution y of (1.6)-(1.9) with $y_{0}=0$ satisfies $y(., T)=y_{1}$. Furthermore, $y \in G^{1,3}([-1,0] \times[0, T])$.

Remark 1.1. (1) As for the heat equation, it is likely that any reachable state for the linear Kortewegde Vries equation can be extended as an holomorphic function on some open set in $\mathbb{C}$.

(2) The reachable states corresponding to the controllability to the trajectories are in $G^{\frac{1}{2}}([-1,0])$, so that they can be extended as functions in $H(\mathbb{C})$. By contrast, the reachable functions in Theorem 1.2 need not be holomorphic on the whole set $\mathbb{C}$ : they can have poles outside $D(0, R)$.

\footnotetext{
${ }^{1}$ It is conjectured that it belongs to $G^{\frac{1}{3}}([-1,0])$.
} 
(3) The set $\mathcal{R}_{R}$ takes a very simple form when $a=0$. Indeed, in that case

$$
\begin{gathered}
\mathcal{R}_{R}=\left\{y \in C([-1,0]) ; \exists z \in H(D(0, R)), y=z_{\mid[-1,0]} \text { and } \partial_{x}^{3 n} y(0)=\partial_{x}^{3 n+1} y(0)=0 \quad \forall n \in \mathbb{N}\right\}, \\
=\left\{y \in C([-1,0]) ; \exists\left(a_{n}\right)_{n \in \mathbb{N}} \in \mathbb{R}^{\mathbb{N}}, \sum_{n=0}^{\infty}\left|a_{n}\right| r^{3 n}<\infty \forall r \in(0, R)\right. \text { and } \\
\left.y(x)=\sum_{n=0}^{\infty} a_{n} x^{3 n+2} \forall x \in[-1,0]\right\} .
\end{gathered}
$$

Note that $y(-1)$ needs not be 0 for $y \in \mathcal{R}_{R}$. Examples of functions in $\mathcal{R}_{R}$ include $(i)$ the polynomial functions of the form $y(x)=\sum_{n=0}^{N} a_{n} x^{3 n+2}$; (ii) the entire function

$$
y(x)=e^{x}+j e^{j x}+j^{2} e^{j^{2} x}
$$

where $j:=e^{i \frac{2 \pi}{3}}$. Note that $y$ is real-valued and $y(-1)>0$ (see Fig. 1).

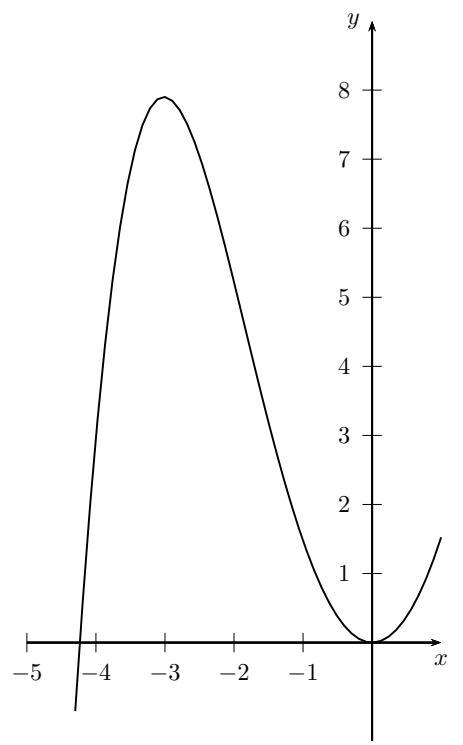

Fig. 1. The function $y(x)=e^{x}+j e^{j x}+j^{2} e^{j^{2} x}$.

Combining Theorem 1.1 and Theorem 1.2, we obtain the following result which implies the exact controllability of (1.6)-(1.9) in $\mathcal{R}_{R}$ for $R>R_{0}$.

Corollary 1.1. Let $a \in \mathbb{R}_{+}, T>0, R>R_{0}, y_{0} \in L^{2}(-1,0)$ and $y_{1} \in \mathcal{R}_{R}$. Then there exists $u \in G^{3}([0, T])$ such that the solution of (1.6)-(1.9) satisfies $y(., T)=y_{1}$.

Since system (1.6)-(1.9) is linear, it is sufficient to pick $u=u_{1}+u_{2}$ where $u_{1}$ is the control given by Theorem 1.1 for $y_{0}$ and $u_{2}$ is the control given by Theorem 1.2 for $y_{1}$.

The paper is outlined as follows. Section 2 is devoted to the null controllability of the linear KdV equation. The flatness property is established in Proposition 2.1. The smoothing effect for the linear $\mathrm{KdV}$ equation from $L^{2}(-1,0)$ to $G^{\frac{1}{2}}([-1,0])$ is derived in Proposition 2.2. The section ends with the proof of Theorem 1.1. Section 3 is concerned with the study of the reachable states. The flatness property is extended to the limit case $s=3$ in Proposition 3.1. Theorem 1.2 then follows from Proposition 3.1 and some version of Borel theorem borrowed from [11]. 


\section{NULl CONTROLLABILITY BY THE FLATNESS APPROACH}

In this section, we are concerned with the null controllability of (1.6)-(1.9).

2.1. Flatness property. Our first task is to establish the flatness property, namely the fact that the solution of (1.6)-(1.8) can be parameterized by the "flat ouput" $\partial_{x}^{2} y(0,$.$) . More precisely, we consider the$ ill-posed system

$$
\begin{aligned}
\partial_{t} y+\partial_{x}^{3} y+a \partial_{x} y=0, & x \in(-1,0), t \in(0, T), \\
y(0, t)=\partial_{x} y(0, t)=0, & t \in(0, T), \\
\partial_{x}^{2} y(0, t)=z(t), & t \in(0, T),
\end{aligned}
$$

and we prove that it admits a solution $y \in G^{\frac{s}{3}, s}([-1,0] \times[0, T])$ whenever $z \in G^{s}([0, T])$ and $1 \leq s<3$.

The trajectory $y$ and the control input $u$ can be written as

$$
\begin{aligned}
y(x, t) & =\sum_{i \geq 0} g_{i}(x) z^{(i)}(t), \\
u(t)=y(-1, t) & =\sum_{i \geq 0} g_{i}(-1) z^{(i)}(t)
\end{aligned}
$$

where the generating functions $g_{i}, i \geq 0$, are defined as in [10]. More precisely, the function $g_{0}$ is defined as the solution of the Cauchy problem

$$
\begin{aligned}
g_{0}^{\prime \prime \prime}(x)+a g_{0}^{\prime}(x) & =0, \quad x \in(-1,0), \\
g_{0}(0)=g_{0}^{\prime}(0) & =0, \\
g_{0}^{\prime \prime}(0) & =1
\end{aligned}
$$

(where ${ }^{\prime}=d / d x$ ), while the function $g_{i}$ for $i \geq 1$ is defined inductively as the solution of the Cauchy problem

$$
\begin{aligned}
g_{i}^{\prime \prime \prime}(x)+a g_{i}^{\prime}(x) & =-g_{i-1}(x), \quad x \in(-1,0), \\
g_{i}(0)=g_{i}^{\prime}(0)=g_{i}^{\prime \prime}(0) & =0 .
\end{aligned}
$$

It is well known that $g_{i}$ for $i \geq 1$ can be expressed in terms of $g_{0}$ and $g_{i-1}$ as

$$
g_{i}(x)=-\int_{0}^{x} g_{0}(x-\xi) g_{i-1}(\xi) d \xi .
$$

Remark 2.1. (1) If $a=0$, then it follows from direct integrations of (2.6)-(2.8) and (2.9)-(2.10) that

$$
g_{i}(x)=(-1)^{i} \frac{x^{3 i+2}}{(3 i+2) !}, \quad x \in[-1,0], i \geq 0 .
$$

(2) If $a>0$, then the solution of (2.6)-(2.8) reads

$$
g_{0}(x)=\frac{1}{a}(1-\cos (\sqrt{a} x)) .
$$

To ensure the convergence of the series in (2.4), we first have to establish some estimates for $\left\|g_{i}\right\|_{L^{\infty}(-1,0)}$.

Lemma 2.1. Let $a \in \mathbb{R}_{+}$. Then for all $i \geq 0$

$$
\left|g_{i}(x)\right| \leq \frac{|x|^{3 i+2}}{(3 i+2) !} \quad \forall x \in[-1,0] .
$$


Proof. If $a=0$, then (2.14) is a direct consequence of (2.12). Assume now that $a>0$ and let us prove (2.14) by induction on $i$. It follows from (2.13) that

$$
0 \leq g_{0}(x) \leq \frac{x^{2}}{2}, \quad \forall x \in[-1,0],
$$

so that (2.14) is true for $i=0$. Assume now that (2.14) is true for some $i-1 \geq 0$. Then, integrating by parts twice in (2.11) and using (2.7), we see that

$$
\begin{aligned}
g_{i}(x) & =-\underbrace{\left[g_{0}(x-\xi) \int_{0}^{\xi} g_{i-1}(\zeta) d \zeta\right]_{0}^{x}}_{=0}-\int_{0}^{x} g_{0}^{\prime}(x-\xi)\left(\int_{0}^{\xi} g_{i-1}(\zeta) d \zeta\right) d \xi \\
& =-\underbrace{\left[g_{0}^{\prime}(x-\xi) \int_{0}^{\xi}\left(\int_{0}^{\zeta} g_{i-1}(\sigma) d \sigma\right) d \zeta\right]_{0}^{x}}_{=0}-\int_{0}^{x} \underbrace{g_{0}^{\prime \prime}(x-\xi)}_{\cos (\sqrt{a}(x-\xi))}\left(\int_{0}^{\xi}\left(\int_{0}^{\zeta} g_{i-1}(\sigma) d \sigma\right) d \zeta\right) d \xi .
\end{aligned}
$$

It follows that

$$
\left|g_{i}(x)\right| \leq\left|\int_{0}^{x}\left(\int_{0}^{\xi}\left(\int_{0}^{\zeta}\left|g_{i-1}(\sigma)\right| d \sigma\right) d \zeta\right) d \xi\right| \leq\left|\int_{0}^{x}\left(\int_{0}^{\xi}\left(\int_{0}^{\zeta} \frac{\sigma^{3 i-1}}{(3 i-1) !} d \sigma\right) d \zeta\right) d \xi\right|=\frac{|x|^{3 i+2}}{(3 i+2) !}
$$

as desired.

We are now in a position to solve system (2.1)-(2.3).

Proposition 2.1. Let $s \in[1,3), z \in G^{s}([0, T])$, and $y=y(x, t)$ be as in (2.4). Then $y \in G^{\frac{s}{3}, s}([-1,0] \times[0, T])$ and it solves (2.1)-(2.3).

Proof. We need to estimate the behavior of the constants in the equivalence of norms in $W^{n, p}([-1,0])$ as $n \rightarrow \infty$. For $n \in \mathbb{N}, p \in[1, \infty]$, and $f \in W^{n, p}(-1,0)$, we denote $\|f\|_{p}=\|f\|_{L^{p}(-1,0)}$ and

$$
\|f\|_{n, p}=\sum_{i=0}^{n}\left\|\partial_{x}^{i} f\right\|_{p}
$$

The following result will be used several times. Its proof is given in appendix, for the sake of completeness.

Lemma 2.2. Let $p \in[1, \infty]$ and $P=\partial_{x}^{3}+a \partial_{x}$, where $a \in \mathbb{R}_{+}$. Then there exists a constant $K=K(p, a)>0$ such that for all $n \in \mathbb{N}$,

$$
\left(1+\frac{1}{a}\right)^{-1}(1+a)^{-n} \sum_{i=0}^{n}\left\|P^{i} f\right\|_{p} \leq\|f\|_{3 n, p} \leq K^{n} \sum_{i=0}^{n}\left\|P^{i} f\right\|_{p}, \quad \forall f \in W^{3 n, p}(-1,0) .
$$

We follow closely [8]. Pick any $z \in G^{s}([0, T])$ for some $s \in[0,3)$. We can find some numbers $M>0$ and $R<1$ such that

Pick any $m, n \in \mathbb{N}$. Then

$$
\left|z^{(i)}(t)\right| \leq M \frac{(i !)^{s}}{R^{i}}, \quad \forall i \in \mathbb{N}, \forall t \in[0, T]
$$

$$
\partial_{t}^{m} P^{n}\left(g_{i}(x) z^{(i)}(t)\right)= \begin{cases}z^{(i+m)}(t)(-1)^{n} g_{i-n}(x) & \text { if } i-n \geq 0, \\ 0 & \text { if } i-n<0 .\end{cases}
$$


Assume that $i \geq n$. Setting $j=i-n$ and $N=n+m$, so that $j+N=i+m$, we have that

$$
\left|\partial_{t}^{m} P^{n}\left(g_{i}(x) z^{(i)}(t)\right)\right| \leq M \frac{(i+m) !^{s}}{R^{i+m}} \frac{1}{(3(i-n)+2) !} \leq M \frac{(j+N) !^{s}}{R^{j+N}} \frac{1}{(3 j+2) !} .
$$

Let

$$
S:=\sum_{i \geq n}\left|\partial_{t}^{m} P^{n}\left(g_{i}(x) z^{(i)}(t)\right)\right| .
$$

Using the classical estimate $(j+N) ! \leq 2^{j+N} j ! N !$ and the equivalence $(3 j) ! \sim 3^{3 j+\frac{1}{2}}(2 \pi j)^{-1}(j !)^{3}$ which follows at once from Stirling formula, we obtain that

$$
\begin{aligned}
S & \leq M \sum_{j \geq 0} \frac{(j+N) !^{s}}{R^{j+N}} \frac{1}{(3 j+2) !} \\
& \leq M^{\prime} \sum_{j \geq 0} \frac{\left(2^{j+N} j ! N !\right)^{s}}{R^{j+N}} \frac{2 \pi(j+1)}{(3 j+2)(3 j+1) 3^{3 j+\frac{1}{2}}(j !)^{3}} \\
& \leq M^{\prime \prime} \frac{(N !)^{s}}{\left(\frac{R}{2^{s}}\right)^{N}}
\end{aligned}
$$

for some positive constants $M^{\prime}, M^{\prime \prime}$. Indeed, since $s<3$, we have that

$$
\sum_{j \geq 0} \frac{\left(2^{j} j !\right)^{s}}{R^{j}} \frac{2 \pi(j+1)}{(3 j+2)(3 j+1) 3^{3 j+\frac{1}{2}}(j !)^{3}}<+\infty .
$$

Using again the fact that $N !=(n+m) ! \leq 2^{n+m} n ! m !$, we arrive to

$$
S \leq M^{\prime \prime} \frac{n !^{s} m !^{s}}{R_{2}^{n} R_{1}^{m}}
$$

with $R_{1}=R_{2}=R / 2^{s}$. Let $K$ be as in Lemma 2.2 for $p=\infty$. Then we have

$$
\begin{aligned}
\sum_{i \geq n}\left\|\partial_{t}^{m}\left(g_{i}(x) z^{(i)}(t)\right)\right\|_{3 n, \infty} & \leq K^{n} \sum_{i \geq n} \sum_{0 \leq j \leq n}\left\|\partial_{t}^{m} P^{j}\left(g_{i}(x) z^{(i)}(t)\right)\right\|_{\infty} \\
& \leq M^{\prime \prime} K^{n} \sum_{0 \leq j \leq n}\left(\frac{j !^{s} m !^{s}}{R_{2}^{j} R_{1}^{m}}\right) \\
& \leq M^{\prime \prime \prime} \frac{n !^{s}}{R_{2}^{\prime n}} \frac{m !^{s}}{R_{1}^{m}}
\end{aligned}
$$

for some $R_{2}^{\prime}>0$ and some $M^{\prime \prime \prime}>0$. This shows that the series of derivatives $\partial_{t}^{m} \partial_{x}^{l}\left(g_{i}(x) z^{(i)}(t)\right)$ is uniformly convergent on $[-1,0] \times[0, T]$ for all $m, l \in \mathbb{N}$, so that $y \in C^{\infty}([-1,0] \times[0, T])$ and it satisfies for $l \leq 3 n$

$$
\left|\partial_{t}^{m} \partial_{x}^{l} y(x, t)\right| \leq M^{\prime \prime \prime} \frac{n !^{s}}{R_{2}^{\prime n}} \frac{m !^{s}}{R_{1}^{m}} \quad \forall x \in[-1,0], \forall t \in[0, T]
$$

for some constant $M^{\prime \prime \prime}>0$. Note that

$$
n !^{s} \sim\left(\frac{2 \pi n(3 n) !}{3^{3 n+\frac{1}{2}}}\right)^{\frac{s}{3}}
$$


It follows that if $l \in\{3 n-2,3 n-1,3 n\}$, then $n !^{s} / R_{2}^{\prime n} \leq C^{\prime} l !^{\frac{s}{3}} / R_{2}^{\prime \prime l}$ for some $C^{\prime}>0$ and $R_{2}^{\prime \prime}>0$. This yields

$$
\left|\partial_{t}^{m} \partial_{x}^{l} y(x, t)\right| \leq C^{\prime} M^{\prime \prime \prime} \frac{m !^{s}}{R_{1}^{m}} \frac{l !^{\frac{s}{3}}}{R_{2}^{\prime \prime l}}, \quad \forall x \in[-1,0], \forall t \in[0, T]
$$

as desired. The fact that $y$ solves (2.1)-(2.3) is obvious.

2.2. Smoothing effect. We now turn our attention to the smoothing effect. We show that any solution $y$ of the initial value problem (1.6)-(1.9) with $u \equiv 0$ and $y_{0} \in L^{2}(-1,0)$ is a function Gevrey of order $1 / 2$ in $x$ and $3 / 2$ in $t$ for $t>0$.

Proposition 2.2. Let $y_{0} \in L^{2}(-1,0)$ and $u(t)=0$ for $t \in \mathbb{R}_{+}$. Then the solution $y$ of (1.6)-(1.9) satisfies $y \in G^{\frac{1}{2}, \frac{3}{2}}([-1,0] \times[\varepsilon, T])$ for all $0<\varepsilon<T<\infty$. More precisely, there exist some positive constant $K, R_{1}, R_{2}$ such that

$$
\left|\partial_{t}^{n} \partial_{x}^{p} y(x, t)\right| \leq K t^{-\frac{3 n+p+3}{2}} \frac{n !^{\frac{3}{2}}}{R_{1}^{n}} \frac{p !^{\frac{1}{2}}}{R_{2}^{p}} \quad \forall p, n \in \mathbb{N}, \forall t \in(0, T], \forall x \in[-1,0] .
$$

Proof. Using (2.18) on intervals of length one, we can, without loss of generality, assume that $T=1$.

Let us introduce the operator $A y=-P y=-\partial_{x}^{3} y-a \partial_{x} y$ with domain

$$
D(A)=\left\{y \in H^{3}(-1,0) ; y(-1)=y(0)=\partial_{x} y(0)=0\right\} \subset L^{2}(-1,0) .
$$

Then it follows from [15] that $A$ generates a semigroup of contractions in $L^{2}(-1,0)$, and tht a global Kato smoothing effect holds. More precisely, if $y=e^{t A} y_{0}$ is the mild solution issuing from $y_{0}$ at $t=0$, then we have for all $T>0$

$$
\begin{aligned}
\|y(T)\|_{L^{2}} & \leq\left\|y_{0}\right\|_{L^{2}} \\
\int_{0}^{T}\left\|\partial_{x} y(., t)\right\|_{L^{2}}^{2} d t & \leq \frac{1}{3}(a T+1)\left\|y_{0}\right\|_{L^{2}}^{2}
\end{aligned}
$$

where $\|f\|_{L^{2}}=\left(\int_{-1}^{0}|f(x)|^{2} d x\right)^{\frac{1}{2}}$. For simplicity, we denote $\|f\|_{H^{p}}=\left(\sum_{i=0}^{p}\left\|\partial_{x}^{i} f\right\|_{L^{2}}^{2}\right)^{\frac{1}{2}}$ for $p \in \mathbb{N}$.

For $p \in\{0,1,2,3,4\}$, we introduce the Banach spaces

$X_{0}=L^{2}(-1,0), X_{1}=H_{0}^{1}(-1,0), X_{2}=\left\{y \in H^{2}(-1,0) ; y(-1)=y(0)=\partial_{x} y(0)=0\right\}, X_{3}=D(A)$,

and

$$
X_{4}=\left\{y \in H^{4}(-1,0), y(-1)=y(0)=\partial_{x} y(0)=\partial_{x}^{3} y(-1)=\partial_{x}^{3} y(0)=0\right\},
$$

$X_{p}$ being endowed with the norm $\|\cdot\|_{H^{p}}$ for $p=0, \ldots, 4$.

CLAIM 1 There is a constant $C=C(a)>0$ such that

$$
\|y(., t)\|_{H^{1}} \leq \frac{C}{\sqrt{t}}\left\|y_{0}\right\|_{L^{2}} \quad \forall t \in(0,1] .
$$

To prove Claim 1, we follow closely [14]. Pick any $y_{0} \in X_{3}=D(A)$, we have by a classical property from semigroup theory that $y \in C([0, T], D(A))$, and that $z(., t)=A y(., t)$ satisfies $z(., t)=e^{t A} z(., 0)$. It follows by (2.19) that

$$
\|z(., t)\|_{L^{2}} \leq\|z(., 0)\|_{L^{2}} \quad \forall t \in(0,1]
$$


Summing with (2.19), this yields

$$
\|y(., t)\|_{L^{2}}+\|P y(., t)\|_{L^{2}} \leq\|y(., t)\|_{L^{2}}+\left\|P y_{0}\right\|_{L^{2}}, \quad t \in(0,1] .
$$

Using Lemma 3.2, this yields

$$
\left\|y_{0}\right\|_{H^{3}} \leq C_{3}\left\|y_{0}\right\|_{H^{3}}, \quad t \in(0,1]
$$

for some $C_{3}=C_{3}(a)>0$. Using interpolation, and noticing that $x_{1}=\left[X_{0}, X_{3}\right]_{\frac{1}{3}}$, we infer the existence of some constant $C_{1}=C_{1}(a)>0$ such that

$$
\|y(., t)\|_{H^{1}} \leq C_{1}\left\|y_{0}\right\|_{H^{1}} \quad \forall y_{0} \in X_{1}, \forall t \in(0,1] .
$$

This yields for $0<s<t \leq 1\|y(., t)\|_{H^{1}}^{2} \leq C_{1}^{2}\|y(., s)\|_{H^{1}}^{2}$, which gives upon integration over $(0, t)$ for $t \in(0,1]$

$$
t\|y(., t)\|_{H^{1}}^{2} \leq C_{1}^{2} \int_{0}^{t}\|y(., s)\|_{H^{1}}^{2} d s \leq \frac{C_{1}^{2}}{3}((a+3) T+1)\left\|y_{0}\right\|_{L^{2}}^{2}
$$

where we used (2.19)-(2.20). Thus (2.21) holds. The proof of Claim 1 is achieved.

Claim 2. There is some constant $C>0$ such that

$$
\|y(., t)\|_{H^{p+1}} \leq \frac{C}{\sqrt{t}}\left\|y_{0}\right\|_{H^{p}}, \quad \text { for } p \in\{0,1,2,3\}, y \in X_{p}, t \in(0,1] .
$$

To prove Claim 2, we pick again $y_{0} \in D(A)$ and set $z(., t)=A y(., t)$. We infer from (2.21) applied to $z(., t)$ that

$$
\|A y(., t)\|_{H^{1}}=\|z(., t)\|_{H^{1}} \leq \frac{C}{\sqrt{t}}\|z(., 0)\|_{H^{1}}=\frac{C}{\sqrt{t}}\left\|A y_{0}\right\| .
$$

Combined with (2.21), this gives

$$
\|y(., t)\|_{H^{1}}+\left\|\left(\partial_{x}^{3}+a \partial_{x}\right) y(., t)\right\|_{H^{1}} \leq \frac{C}{\sqrt{t}}\left(\left\|y_{0}\right\|_{L^{2}}+\left\|A y_{0}\right\|_{L^{2}}\right) .
$$

We know from Lemma 3.2 that for $z \in H^{3}(-1,0)$

$$
\|z\|_{H^{3}} \leq C\left(\|z\|_{L^{2}}+\|P z\|_{L^{2}}\right) .
$$

It follows that for $z \in X_{4}(C$ denoting a positive constant that may change from line to line, and that do not depend on $t$ and on $y_{0}$ )

$$
\begin{aligned}
\|z\|_{H^{4}} & \leq C\left(\|z\|_{H^{3}}+\left\|\partial_{x}^{4} z\right\|_{L^{2}}\right) \\
& \leq C\left(\|z\|_{L^{2}}+\|P z\|_{L^{2}}+\left\|\partial_{x}\left(\partial_{x}^{3} z+a \partial_{x} z\right)\right\|_{L^{2}}+a\left\|\partial_{x}^{2} z\right\|_{L^{2}}\right) \\
& \leq C\left(\|z\|_{H^{1}}+\|A z\|_{H^{1}}\right) .
\end{aligned}
$$

Combined with (2.25), this gives

$$
\|y(., t)\|_{H^{4}} \leq \frac{C}{\sqrt{t}}\left\|y_{0}\right\|_{H^{3}} \quad \forall t \in(0,1]
$$

for all $y_{0} \in X_{4}$, and also for all $y_{0} \in X_{3}=D(A)$ by density.

Interpolating between (2.21) and (2.26), we obtain (2.24). The proof of Claim 2 is achieved. 
Using Claim 2 inductively and spitting $[0, t]$ into $[0, t / 3] \cup[t / 3,2 t / 3] \cup[2 t / 3, t]$, we infer that

$$
\begin{aligned}
& \left\|a \partial_{x} y(., t)\right\|_{L^{2}} \leq \frac{C a}{\sqrt{t}}\left\|y_{0}\right\|_{L^{2}} \quad t \in(0,1] \\
& \left\|\partial_{x}^{3} y(., t)\right\|_{L^{2}} \leq \frac{C}{\sqrt{\frac{t}{3}}}\left\|\partial_{x}^{2} y\left(., \frac{2 t}{3}\right)\right\|_{L^{2}} \leq\left(\frac{C}{\sqrt{\frac{t}{3}}}\right)^{2}\left\|\partial_{x} y\left(., \frac{t}{3}\right)\right\|_{L^{2}} \leq\left(\frac{C}{\sqrt{\frac{t}{3}}}\right)^{3}\left\|y_{0}\right\|_{L^{2}}
\end{aligned}
$$

Combining (2.27) and (2.28), we infer the existence of a constant $C^{\prime}=C^{\prime}(a)>0$ (say $C^{\prime} \geq 1$, for simplicity), such that

$$
\|A y(t)\|_{L^{2}} \leq \frac{C^{\prime}}{t^{\frac{3}{2}}}\left\|y_{0}\right\|_{L^{2}}, \quad \text { for } y_{0} \in L^{2}(-1,-), t \in(0,1] .
$$

For $y_{0} \in D\left(A^{n-1}\right), z(t)=A^{n-1} y(t)$ satisfies $z(., t)=e^{t A}\left(A^{n-1} y_{0}\right)$ and thus

$$
\left\|A^{n} y(., t)\right\|_{L^{2}}=\|A z(., t)\|_{L^{2}} \frac{C^{\prime}}{t^{\frac{3}{2}}}\left\|A^{n-1} y_{0}\right\| .
$$

For $y_{0} \in L^{2}(-1,0)$ and $t \in(0,1]$, splitting $[0, t]$ into $\left[0, \frac{t}{n}\right] \cup\left[\frac{t}{n}, \frac{2 t}{n}\right] \cup \cdots \cup\left[\frac{n-1}{n} t, t\right]$, we obtain

$$
\left\|A^{n} y(., t)\right\|_{L^{2}} \leq \frac{C^{\prime}}{\left(\frac{t}{n}\right)^{\frac{3}{2}}}\left\|A^{n-1} y\left(\frac{n-1}{n} t\right)\right\| \leq \cdots \leq\left(\frac{C^{\prime}}{\left(\frac{t}{n}\right)^{\frac{3}{2}}}\right)^{n}\left\|y_{0}\right\|=\frac{C^{\prime n}}{t^{\frac{3 n}{2}}} n^{\frac{3 n}{2}}\left\|y_{0}\right\|_{L^{2}} .
$$

If $p \in \mathbb{N}$ is given, we pick $n \in \mathbb{N}$ such that $3 n-3 \leq p \leq 3 n-1$. Then, by Sobolev embedding, we have that

$$
\begin{aligned}
\left\|\partial_{x}^{p} y(., t)\right\|_{L^{\infty}} & \leq C\|y(., t)\|_{H^{p+1}} \\
& \leq C\|y(., t)\|_{H^{3 n}} \\
& \leq C\left(\|y(., t)\|_{L^{2}}+\|P y(., t)\|_{L^{2}}+\cdots+\left\|P^{n} y(., t)\right\|_{L^{2}}\right) \\
& \leq C\left(1+\frac{C^{\prime}}{t^{\frac{3}{2}}}+\cdots+\frac{C^{\prime n}}{t^{\frac{3 n}{2}}} n^{\frac{3 n}{2}}\right)\left\|y_{0}\right\|_{L^{2}} \\
& \leq C \frac{C^{\prime n}(n+1) n^{\frac{3 n}{2}}}{t^{\frac{3 n}{2}}}\left\|y_{0}\right\|_{L^{2}} .
\end{aligned}
$$

Since

$$
(n+1) \frac{(3 n)^{\frac{3 n}{2}}}{3^{\frac{3 n}{2}}} \leq \frac{\left(1+\frac{p}{3}\right)}{3^{\frac{p+1}{2}}}(p+3)^{\frac{p+3}{2}} \leq C^{\prime \prime} p^{\frac{3}{4}}\left(\frac{e}{3}\right)^{\frac{p}{2}}((p+1) !)^{\frac{1}{2}}
$$

we see that there are some constants $C^{\prime \prime \prime}>0$ and $R>0$ such that

$$
\left|\partial_{x}^{p} y(x, t)\right| \leq \frac{C^{\prime \prime \prime}}{R^{p} t^{\frac{p+3}{2}}}(p !)^{\frac{1}{2}}, \quad p \in \mathbb{N}, t \in(0,1], x \in[0,1] .
$$


From (2.30), we have that $y \in C\left((0,1], D\left(A^{n}\right)\right)$ for all $n \geq 0$ and hence that $y \in C^{\infty}([0,1] \times(0,1])$. Finally, for all $n \geq 0$ and $p \geq 0$, we have that

$$
\begin{aligned}
\partial_{t}^{n} \partial_{x}^{p} y & =(-1)^{n} P^{n} \partial_{x}^{p} y \\
& =(-1)^{n}\left(\partial_{x}^{3}+a \partial_{x}\right)^{n} \partial_{x}^{p} y \\
& =(-1)^{n} \sum_{q=0}^{n}\left(\begin{array}{c}
n \\
q
\end{array}\right) a^{n-q} \partial_{x}^{n+2 q+p} y
\end{aligned}
$$

and hence, assuming $R^{\prime}<1$,

$$
\begin{aligned}
\left|\partial_{t}^{n} \partial_{x}^{p} y(x, t)\right| & \leq C^{\prime \prime} \sum_{q=0}^{n}\left(\begin{array}{c}
n \\
q
\end{array}\right) a^{n-q} \frac{(n+2 q+p) !^{\frac{1}{2}}}{R^{\prime n+2 q+p} t^{\frac{n+2 q+p+3}{2}}} \\
& \leq C^{\prime \prime} \frac{(n+1)(2 a)^{n}(3 n+p) !^{\frac{1}{2}}}{R^{\prime 3 n+p} t^{\frac{3 n+p+3}{2}}} \\
& \leq C^{\prime \prime} \frac{(n+1)(2 a)^{n} 2^{\frac{3 n+p}{2}}(3 n !)^{\frac{1}{2}} p !^{\frac{1}{2}}}{R^{\prime 3 n+p} t^{\frac{3 n+p+3}{2}}} \\
& \leq \frac{K}{t^{\frac{3 n+p+3}{2}}} \frac{n !^{\frac{3}{2}}}{R_{1}^{n}} \frac{p !^{\frac{1}{2}}}{R_{2}^{p}}
\end{aligned}
$$

for some $K, C_{1}, C_{2} \in(0,+\infty)$ and for all $t \in(0,1]$ and all $x \in[0,1]$. The proof of Proposition 2.2 is complete.

It is actually expected that for $y_{0} \in L^{2}(-1,0)$ and $u \equiv 0$, we have that

$$
y \in G^{\frac{1}{3}, 1}([-1,0] \times[\varepsilon, T]) \quad \forall 0<\varepsilon<T<\infty .
$$

Proving such a property seems to be challenging. The smoothing effect from $L^{2}$ to $G^{1 / 3}$ is much easy to establish on $\mathbb{R}$ for data with compact support. The proof of the following result is given in appendix.

Proposition 2.3. Let $y_{0} \in L^{2}(\mathbb{R})$ be such that $y_{0}(x)=0$ for a.e. $x \in \mathbb{R} \backslash[-L, L]$ for some $L>0$. Let $y=y(x, t)$ denote the solution of the Cauchy problem

$$
\begin{aligned}
\partial_{t} y+\partial_{x}^{3} y & =0, \quad t>0, x \in \mathbb{R}, \\
y(x, 0) & =y_{0}(x), \quad x \in \mathbb{R} .
\end{aligned}
$$

Then $y \in G^{\frac{1}{3}, 1}([-l, l] \times[\varepsilon, T])$ for all $l>0$ and all $0<\varepsilon<T$.

2.3. Proof of Theorem 1.1. Pick any $y_{0} \in L^{2}(-1,0), T>0$, and $s \in\left[\frac{3}{2}, 3\right)$. Let $\bar{y}$ denote the solution of the free evolution for the KdV system:

$$
\begin{aligned}
\partial_{t} \bar{y}+\partial_{x}^{3} \bar{y}+a \partial_{x} \bar{y}=0, & x \in(-1,0), t \in(0, T), \\
\bar{y}(0, t)=\partial_{x} \bar{y}(0, t)=\bar{y}(-1, t)=0, & t \in(0, T), \\
\bar{y}(x, 0)=y_{0}(x), & x \in(-1,0) .
\end{aligned}
$$


It follows from Proposition 2.2 that $\bar{y} \in G^{\frac{1}{2}, \frac{3}{2}}([-1,0] \times[\varepsilon, T])$ for any $\varepsilon \in(0, T)$. In particular, $\partial_{x}^{2} \bar{y}(0,.) \in$ $G^{\frac{3}{2}}([\varepsilon, T])$ for any $\varepsilon \in(0, T)$. Pick any $\tau \in(0, T)$ and let

$$
z(t)=\phi_{s}\left(\frac{t-\tau}{T-\tau}\right) \partial_{x}^{2} \bar{y}(0, t)
$$

where $\phi_{s}$ is the "step function"

$$
\phi_{S}(\rho)= \begin{cases}1 & \text { if } \rho \leq 0, \\ 0 & \text { if } \rho \geq 1, \\ \frac{e^{-\frac{M}{(1-\rho)^{\sigma}}}}{e^{-\frac{M}{\rho^{\sigma}}}+e^{-\frac{M}{(1-\rho)^{\sigma}}}} & \text { if } \rho \in(0,1),\end{cases}
$$

with $M>0$ and $\sigma:=(s-1)^{-1}$. As $\phi_{s}$ is Gevrey of order $s$ (see e.g. [12]) and $s \geq 3 / 2$, we infer that $z \in G^{S}([\varepsilon, T])$ for all $\varepsilon \in(0, T)$. Let

$$
y(x, t)= \begin{cases}y_{0}(x) & \text { if } x \in[-1,0], t=0, \\ \sum_{i \geq 0} g_{i}(x) z^{(i)}(t) & \text { if } x \in[-1,0], t \in(0, T] .\end{cases}
$$

Then, by Proposition 2.1, $y \in G^{\frac{s}{3}, s}([-1,0] \times[\varepsilon, T])$ for all $\varepsilon \in(0, T)$, and it satisfies (2.1)-(2.3). Furthermore,

$$
\partial_{x}^{p} y(0, t)=\partial_{x}^{p} \bar{y}(0, t), \quad \forall t \in(0, T), \forall p \in\{0,1,2\},
$$

so that

$$
y(x, t)=\bar{y}(x, t) \quad \forall(x, t) \in[-1,0] \times(0, \tau),
$$

by Holmgren theorem. We infer that $y \in C\left([0, T], L^{2}(-1,0)\right)$ and that it solves (1.6)-(1.9) if we define $u$ as in (2.5). Note that $u(t)=0$ for $0<t<\tau$ and that $u \in G^{s}([0, T])$. Finally $y(., T)=0$, for $z^{(i)}(T)=0$ for all $i \geq 0$. The proof of Theorem 1.1 is complete.

\section{REACHABLE STATES}

3.1. The limit case $s=3$ in the flatness property. The following result extends the flatness property depicted in Proposition 2.1 to the limit case $s=3$.

Proposition 3.1. Assume that $z \in G^{3}([0, T])$ with

$$
\left|z^{(j)}(t)\right| \leq M \frac{(3 j) !}{R^{3 j}} \quad \forall j \geq 0, \forall t \in[0, T]
$$

where $R>1$, and let $y=y(x, t)$ be as in (2.4). Then $y \in G^{1,3}([-1,0] \times[0, T])$ and it solves (2.1)-(2.3).

Proof. We follow closely [11]. Pick any $m, n \in \mathbb{N}$. By (2.17), we can assume that $i \geq n$. Setting $j=$ $3 i-3 n$ and $N=3 n+3 m$, so that $j+N=3 i+3 m$, we have that

$$
\left|\partial_{t}^{m} P^{n}\left(g_{i}(x) z^{(i)}(t)\right)\right| \leq M \frac{(3 i+3 m) !}{R^{3 i+3 m}} \frac{1}{(3(i-n)+2) !} \leq M \frac{(j+N) !}{R^{j+N}} \frac{1}{(j+2) !} .
$$

Let

$$
S:=\sum_{i \geq n}\left|\partial_{t}^{m} P^{n}\left(g_{i}(x) z^{(i)}(t)\right)\right|
$$


If $N \leq 2$, then $S \leq M \sum_{j \geq 0} R^{-(j+N)}<\infty$ for $R>1$. Assume from now on that $N \geq 2$. Then

$$
\begin{aligned}
S & \leq M \sum_{j \geq 0} \frac{(j+3) \cdots(j+N)}{R^{j+N}} \\
& \leq M \sum_{k \geq 0} \sum_{k N \leq j<(k+1) N} \frac{(j+3) \cdots(j+N)}{R^{j+N}} \\
& \leq M \sum_{k \geq 0} N \frac{((k+2) N)^{N-2}}{R^{(k+1) N}} \\
& \leq M N^{N-1} \sum_{k \geq 0}\left(\frac{k+2}{R^{k+1}}\right)^{N} .
\end{aligned}
$$

Pick any $\sigma \in(0,1)$ and let $a:=\sup _{k \geq 0} \frac{k+2}{\left(R^{1-\sigma}\right)^{k+1}}<\infty$. We infer from [11, Proof of Proposition 3.1] that

$$
\sum_{k \geq 0}\left(\frac{k+2}{R^{k+1}}\right)^{N} \leq \frac{a^{N}}{R^{N \sigma}-1}
$$

so that

$$
S \leq M N^{N-1} \frac{a^{N}}{R^{N \sigma}-1} \leq M^{\prime}\left(\frac{a e}{R^{\sigma}}\right)^{N} \frac{N !}{N^{\frac{3}{2}}}
$$

for some constant $M^{\prime}>0$, by using Stirling formula. Next, we have that

$$
N !=(3 n+3 m) ! \leq 2^{3 n+3 m}(3 n) !(3 m) !
$$

and using again the estimate

$$
\frac{(3 m) !}{m !^{3}} \sim 3^{3 m} \frac{\sqrt{3}}{2 \pi m}
$$

we infer that

$$
\begin{aligned}
S & \leq M^{\prime \prime}\left(\frac{a e}{R^{\sigma}}\right)^{3 m+3 n} 2^{3 n+3 m}(3 n) !\left(\frac{3^{3 m}}{m+1}(m !)^{3}\right) \frac{1}{(m+n+1)^{\frac{3}{2}}} \\
& \leq M^{\prime \prime \prime} \frac{(3 n) !}{R_{2}^{3 n}} \frac{(m !)^{3}}{R_{1}^{m}} \frac{1}{(n+1)^{\frac{3}{2}}}
\end{aligned}
$$

for some positive constants $M^{\prime \prime}, M^{\prime \prime \prime}, R_{1}$ and $R_{2}$. There is no loss of generality in assuming that $R_{2}<1$. Let $K$ be as in Lemma 2.2 for $p=\infty$. Then we have

$$
\begin{aligned}
\sum_{i \geq n}\left\|\partial_{t}^{m}\left(g_{i}(x) z^{(i)}(t)\right)\right\|_{3 n, \infty} & \leq K^{n} \sum_{i \geq n} \sum_{0 \leq j \leq n}\left\|\partial_{t}^{m} P^{j}\left(g_{i}(x) z^{(i)}(t)\right)\right\|_{\infty} \\
& \leq M^{\prime \prime \prime} K^{n} \sum_{0 \leq j \leq n}\left(\frac{(m !)^{3}}{R_{1}^{m}} \frac{(3 j) !}{R_{2}^{3 j}} \frac{1}{(j+1)^{\frac{3}{2}}}\right) \\
& \leq M^{\prime \prime \prime} K^{n} \frac{(3 n) !}{R_{2}^{3 n}} \frac{(m !)^{3}}{R_{1}^{m}} \sum_{j \geq 0} \frac{1}{(j+1)^{\frac{3}{2}}} .
\end{aligned}
$$


This shows that the series of derivatives $\partial_{t}^{m} \partial_{x}^{l}\left(g_{i}(x) z^{(i)}(t)\right)$ is uniformly convergent on $[-1,0] \times[0, T]$ for all $m, l \in \mathbb{N}$, so that $y \in C^{\infty}([-1,0] \times[0, T])$, and that the function $y$ satisfies for $l \leq 3 n$

$$
\left|\partial_{t}^{m} \partial_{x}^{l} y(x, t)\right| \leq M^{\prime \prime \prime \prime} K^{n} \frac{(3 n) !}{R_{2}^{3 n}} \frac{(m !)^{3}}{R_{1}^{m}} \quad \forall x \in[-1,0], \forall t \in[0, T] .
$$

for some constant $M^{\prime \prime \prime \prime \prime}>0$. Finally, if $l \in\{3 n-2,3 n-1,3 n\}$, then $(3 n) !\left(K / R_{2}^{3}\right)^{n} \leq C^{\prime} l ! / R_{2}^{\prime l}$ for some $C^{\prime}>0, R_{2}^{\prime}>0$. This yields

$$
\left|\partial_{t}^{m} \partial_{x}^{l} y(x, t)\right| \leq C^{\prime} M^{\prime \prime \prime \prime} \frac{(m !)^{3}}{R_{1}^{m}} \frac{l !}{R_{2}^{\prime l}}, \quad \forall x \in[-1,0], \forall t \in[0, T] .
$$

3.2. Proof of Theorem 1.2. Pick any $R>R_{0}=e^{(3 e)^{-1}}(1+a)^{\frac{1}{3}}$ and any $y_{1} \in \mathcal{R}_{R}$. Our first task is to write $y_{1}$ in the form

$$
y_{1}(x)=\sum_{i \geq 0} b_{i} g_{i}(x), \quad x \in[-1,0] .
$$

Note that if (3.2) holds with a convergence in $W^{n, \infty}(-1,0)$ for all $n \geq 0$, then

$$
\partial_{x}^{2} P^{n} y_{1}(0)=\sum_{i \geq 0} b_{i} \partial_{x}^{2} P^{n} g_{i}(0)=\sum_{i \geq n} b_{i} \partial_{x}^{2} g_{i-n}(0)=b_{n} .
$$

Set

$$
b_{n}:=\partial_{x}^{2} P^{n} y_{1}(0), \quad \forall n \geq 0 .
$$

Since $y_{1} \in \mathcal{R}_{R}$ with $R>R_{0}$, there exists for any $r \in\left(R_{0}, R\right)$ a constant $C=C(r)>0$ such that

$$
\left|\partial_{x}^{n} y_{1}(x)\right| \leq C \frac{n !}{r^{n}}, \quad \forall x \in[-r, 0], \forall n \in \mathbb{N} .
$$

Using Lemma 2.2, we infer that

$$
\left|b_{n}\right|=\left|P^{n} \partial_{x}^{2} y_{1}(0)\right| \leq\left(1+\frac{1}{a}\right)(1+a)^{n}\left\|\partial_{x}^{2} y_{1}\right\|_{3 n, \infty} \leq C^{\prime}(1+a)^{n} \frac{(3 n+2) !}{r^{3 n+2}}
$$

for some $C^{\prime}>0$ and all $n \geq 0$. We need the following version of Borel Theorem, which is a particular case of [11, Proposition 3.6] (with $a_{p}=[3 p(3 p-1)(3 p-2)]^{-1}$ for $p \geq 1$ ).

Proposition 3.2. Let $\left(d_{q}\right)_{q \geq 0}$ be a sequence of real numbers such that

$$
\left|d_{q}\right| \leq C H^{q}(3 q) ! \quad \forall q \geq 0
$$

for some $H>0$ and $C>0$. Then for all $\tilde{H}>e^{e^{-1}} H$, there exists a function $f \in C^{\infty}(\mathbb{R})$ such that

$$
\begin{aligned}
f^{(q)}(0) & =d_{q} \quad \forall q \geq 0, \\
\left|f^{(q)}(x)\right| & \leq C \tilde{H}^{q}(3 q) ! \quad \forall q \geq 0, \forall x \in \mathbb{R} .
\end{aligned}
$$

Since $r>R_{0}$, we can pick two numbers $H \in\left(0, e^{-e^{-1}}\right)$ and $C^{\prime \prime}>0$ such that

$$
\left|b_{n}\right| \leq C^{\prime \prime} H^{n}(3 n) ! \quad \forall n \geq 0 .
$$


By Proposition 3.2, there exists a function $f \in G^{3}([0, T])$ and a number $R>1$ such that

$$
\begin{aligned}
f^{(i)}(T) & =b_{i} \quad \forall i \geq 0 \\
\left|f^{(i)}(t)\right| & \leq C^{\prime \prime} \frac{(3 i) !}{R^{3 i}} \quad \forall i \geq 0, \forall t \in[0, T] .
\end{aligned}
$$

Pick any $\tau \in(0, T)$ and let

$$
g(t)=1-\phi_{2}\left(\frac{t-\tau}{T-\tau}\right) \quad \text { for } t \in[0, T]
$$

Note that $g \in G^{2}([0, T])$ and that $g(T)=1, g^{(i)}(T)=0$ for all $i \geq 1$. Setting

$$
z(t)=g(t) f(t) \quad \forall t \in[0, T]
$$

we have that $z \in G^{3}([0, T])$ and that

$$
\begin{aligned}
z^{(i)}(T) & =b_{i} \quad \forall i \geq 0 \\
z^{(i)}(0) & =0 \quad \forall i \geq 0 \\
\left|z^{(i)}(t)\right| & \leq C^{\prime \prime \prime} \frac{(3 i) !}{R^{3 i}} \quad \forall i \geq 0, \forall t \in[0, T]
\end{aligned}
$$

for some $C^{\prime \prime \prime}>0$. (The fact that the constant $R>0$ in (3.7) is the same as in (3.4) is proved as in [11, Lemma 3.7].) Let $y$ be as in (2.4). Then by Proposition 3.1 we know that $y \in G^{1,3}([-1,0] \times[0, T])$ and that it solves (2.1)-(2.3). Let $u(t)=y(-1, t)$ for $t \in[0, T]$. Then $u \in G^{3}([0, T])$ and $y$ solves (1.6)-(1.9) with $y_{0}=0$, by (3.6). Furthermore, we have by (3.5) that

$$
y(x, T)=\sum_{i \geq 0} g_{i}(x) z^{(i)}(T)=\sum_{i \geq 0} b_{i} g_{i}(x), \quad x \in[-1,0] .
$$

From the proof of Proposition 3.1, we know that for all $l, m \in \mathbb{N}$, the sequence of partial sums of the series $\sum_{i \geq 0} \partial_{t}^{m} \partial_{x}^{l}\left(g_{i}(x) z^{(i)}(t)\right)$ converges uniformly on $[-1,0] \times[0, T]$ to $\partial_{t}^{m} \partial_{x}^{l} y$, and hence for all $n \geq 0$

$$
\begin{aligned}
& P^{n} y(0, T)=\sum_{i \geq 0} b_{i} P^{n} g_{i}(0)=\sum_{i \geq n} b_{i} g_{i-n}(0)=0 \\
& \partial_{x} P^{n} y(0, T)=\sum_{i \geq 0} b_{i} \partial_{x} P^{n} g_{i}(0)=\sum_{i \geq n} b_{i} \partial_{x} g_{i-n}(0)=0 \\
& \partial_{x}^{2} P^{n} y(0, T)=\sum_{i \geq 0} b_{i} \partial_{x}^{2} P^{n} g_{i}(0)=\sum_{i \geq n} b_{i} \partial_{x}^{2} g_{i-n}(0)=b_{n}=\partial_{x}^{2} P^{n} y_{1}(0) .
\end{aligned}
$$

To conclude that

$$
y(x, T)=y_{1}(x), \quad \forall x \in[-1,0],
$$

it is sufficient to prove the following

Claim 3. If $h \in G^{1}([-1,0])$ is such that $P^{n} h(0)=\partial_{x} P^{n} h(0)=\partial_{x}^{2} P^{n} h(0)=0$ for all $n \in \mathbb{N}$, then $h \equiv 0$.

Indeed, we notice that $P^{0}=i d$ and that $P^{n}=\partial_{x}^{3 n}+\cdots, \partial_{x} P^{n}=\partial_{x}^{3 n+1}+\cdots$, and $\partial_{x}^{2} P^{n}=\partial_{x}^{3 n+2}+\cdots$, where $\cdots$ stands for less order derivatives. Then we obtain by induction that $\partial_{x}^{3 n} h(0)=\partial_{x}^{3 n+1} h(0)=$ $\partial_{x}^{3 n+2} h(0)=0$ for all $n \geq 0$, so that $h \equiv 0$. This completes the proof of Claim 3 and of Theorem 1.2. 


\section{APPENDIX}

3.3. Proof of Lemma 2.2. We first need to prove two simple lemmas. We still use the notation $P=$ $\partial_{x}^{3}+a \partial_{x}$.

Lemma 3.1. Let $a \in \mathbb{R}_{+}$and $p \in[1, \infty]$. Then for all $n \in \mathbb{N}$, we have

$$
\left\|P^{n} f\right\|_{p} \leq(1+a)^{n}\|f\|_{3 n, p} \quad \forall f \in W^{3 n, p}(-1,0) .
$$

Proof. The proof is by induction on $n$. For $n=0$, the result is obvious. If it is true at rank $n-1$, then

$$
\left\|P^{n-1}(P f)\right\|_{p} \leq(1+a)^{n-1}\|P f\|_{3 n-3, p} \leq(1+a)^{n-1}\left(\left\|f^{\prime \prime \prime}\right\|_{3 n-3, p}+a\left\|f^{\prime}\right\|_{3 n-3, p}\right) \leq(1+a)^{n}\|f\|_{3 n, p} .
$$

Lemma 3.2. Let $a \in \mathbb{R}_{+}$and $p \in[1, \infty]$. Then there exists a constant $C_{1}=C_{1}(p, a)>0$ such that

$$
\|f\|_{3, p} \leq C_{1}\left(\|f\|_{p}+\|P f\|_{p}\right) \quad \forall f \in W^{3, p}(-1,0) .
$$

Proof. The seminorm

$$
\|f \mid\|:=\|f\|_{p}+\|P f\|_{p}
$$

is clearly a norm in $W^{3, p}(-1,0)$. Let us check that $W^{3, p}(-1,0)$, endowed with the norm \|\|$\cdot\|\|$, is a Banach space. Pick any Cauchy sequence $\left(f_{n}\right)_{n \geq 0}$ for \|\|$\cdot\|\|$. Then

$$
\left\||| f_{m}-f_{n} \mid\right\|=\left\|f_{m}-f_{n}\right\|_{p}+\left\|P f_{m}-P f_{n}\right\|_{p} \rightarrow 0, \quad \text { as } m, n \rightarrow+\infty .
$$

Since $L^{p}(-1,0)$ is a Banach space, there exist $f, g \in L^{p}(-1,0)$ such that

$$
\left\|f_{n}-f\right\|_{p}+\left\|P f_{n}-g\right\|_{p} \rightarrow 0, \quad \text { as } n \rightarrow \infty .
$$

Since $f_{n} \rightarrow f$ in $\mathcal{D}^{\prime}(-1,0), P f_{n} \rightarrow P f$ in $\mathcal{D}^{\prime}(-1,0)$ as well, and $P f=g$. Thus $f^{\prime \prime}=\int g(x) d x-a f \in$ $L^{p}(-1,0)$, and $f \in W^{2, p}(-1,0)$. This yields $f^{\prime} \in W^{1, p}(-1,0)$ and $f^{\prime \prime \prime}=g-a f^{\prime} \in L^{p}(-1,0)$, and hence $f \in W^{3, p}(-1,0)$. Note that (3.10) can be written $\left\|\left|f_{n}-f \|\right| \rightarrow 0\right.$. This proves that $\left(W^{3, p}(-1,0),\|\| \cdot \|||\right)$ is a Banach space. Now, applying the Banach theorem to the identity map from the Banach space $\left(W^{3, p}(-1,0),\|\cdot\|_{3, p}\right)$ to the Banach space $\left(W^{3, p}(-1,0),\||\cdot|\|\right)$, which is linear, continuous, and bijective, we infer that its inverse is continuous; that is, (3.9) holds.

Let us prove Lemma 2.2. We proceed by induction on $n$. Both inequalities in (2.16) are obvious for $n=0$. Assume now that both inequalities in (2.16) are satisfied up to the rank $n-1$. Let us first prove the left inequality in (2.16) at the rank $n$. Pick any $f \in W^{3 n, p}(-1,0)$. Then, by the induction hypothesis and Lemma 3.1, we obtain

$$
\begin{aligned}
\sum_{i=0}^{n}\left\|P^{i} f\right\|_{p} & =\sum_{i=0}^{n-1}\left\|P^{i} f\right\|_{p}+\left\|P^{n} f\right\|_{p} \\
& \leq\left(1+\frac{1}{a}\right)(1+a)^{n-1}\|f\|_{3 n-3, p}+(1+a)^{n}\|f\|_{3 n, p} \\
& \leq\left(1+\frac{1}{a}\right)(1+a)^{n}\|f\|_{3 n, p},
\end{aligned}
$$

as desired. 
For the right inequality in (2.16), we write

$$
\begin{aligned}
\|f\|_{3 n, p} & =\|f\|_{3 n-3, p}+\left\|\partial_{x}^{3 n-2} f\right\|_{p}+\left\|\partial_{x}^{3 n-1} f\right\|_{p}+\left\|\partial_{x}^{3 n} f\right\|_{p} \\
& \leq K^{n-1} \sum_{i=0}^{n-1}\left\|P^{i} f\right\|_{p}+\left\|\partial_{x}^{3 n-3} f\right\|_{3, p} .
\end{aligned}
$$

But it follows from Lemma 3.2 that

$$
\begin{aligned}
\left\|\partial_{x}^{3 n-3} f\right\|_{3, p} & \leq C_{1}\left(\left\|\partial_{x}^{3 n-3} f\right\|_{p}+\left\|P \partial_{x}^{3 n-3} f\right\|_{p}\right) \\
& \leq C_{1}\left(\left\|\partial_{x}^{3 n-3} f\right\|_{p}+\left\|\partial_{x}^{3 n-3} P f\right\|_{p}\right) \\
& \leq C_{1}\left(\|f\|_{3 n-3, p}+\|P f\|_{3 n-3, p}\right) \\
& \leq C_{1} K^{n-1}\left(\sum_{i=0}^{n-1}\left\|P^{i} f\right\|_{p}+\sum_{i=1}^{n}\left\|P^{i} f\right\|_{p}\right) \\
& \leq 2 C_{1} K^{n-1} \sum_{i=0}^{n}\left\|P^{i} f\right\|_{p} .
\end{aligned}
$$

Combined with (3.11), this yields

$$
\|f\|_{3 n, p} \leq\left(1+2 C_{1}\right) K^{n-1} \sum_{i=0}^{n}\left\|P^{i} f\right\|_{p}
$$

It is sufficient to pick $K:=1+2 C_{1}$.

3.4. Proof of Proposition 2.3. Let Ai denote the Airy function defined as the inverse Fourier transform of $\xi \rightarrow \exp \left(i \xi^{3} / 3\right)$. Then it is well known (see e.g. [7]) that Ai is an entire (i.e. complex analytic on $\mathbb{C}$ ) function satisfying

$$
\operatorname{Ai}^{\prime \prime}(x)=x \operatorname{Ai}(x), \quad \forall x \in \mathbb{C} .
$$

To prove that $y \in G^{\frac{1}{3}, 1}([-l, l] \times[\varepsilon, T])$, we need first check that the Airy function is itself Gevrey of order $1 / 3$.

Claim 4. Ai $\in G^{\frac{1}{3}}([-l, l])$ for any $l>0$.

Let us prove Claim 4. Differentiating $k+1$ times in (3.12) and letting $x=0$ results in

$$
\mathrm{Ai}^{(3+k)}(0)=\left(\mathrm{Ai}^{\prime \prime}\right)^{(k+1)}(0)=(k+1) \mathrm{Ai}^{(k)}(0) \quad \forall k \in \mathbb{N} .
$$

Note that $\mathrm{Ai}^{\prime \prime}(0)=0$ by (3.12) and that

$$
\operatorname{Ai}(0)=\left[3^{\frac{2}{3}} \Gamma\left(\frac{2}{3}\right)\right]^{-1} \neq 0, \quad \operatorname{Ai}^{\prime}(0)=-\left[3^{\frac{1}{3}} \Gamma\left(\frac{1}{3}\right)\right]^{-1} \neq 0 .
$$

We infer that for all $k \in \mathbb{N}$

$$
\mathrm{Ai}^{(3 k)}(0)=\mathrm{Ai}(0) \prod_{j=1}^{k-1}(1+3 j), \quad \mathrm{Ai}^{(3 k+1)}(0)=\mathrm{Ai}^{\prime}(0) \prod_{j=1}^{k-1}(2+3 j), \quad \mathrm{Ai}^{(3 k+2)}(0)=0 .
$$

As it was noticed in [10, Remark 2.7], we have

$$
s^{i} i ! \leq \prod_{j=1}^{i}(r+j s) \leq s^{i}(i+1) ! \quad \forall s \in \mathbb{N}, \forall r \in[0, s] .
$$


Thus there is some constant $C_{1}>0$ such that

$$
\left|\mathrm{Ai}^{(3 k+q)}(0)\right| \leq C_{1} 3^{k} k ! \quad \forall k \geq 0, \forall q \in\{0,1,2\} .
$$

From Stirling formula we have $(3 k) ! \sim \frac{\sqrt{3}}{2 \pi k}\left(3^{k} k !\right)^{3}$ so that

$$
\left|\mathrm{Ai}^{(n)}(0)\right| \leq C_{2}[(n+1) !]^{\frac{1}{3}} \leq C_{3} \frac{n !^{\frac{1}{3}}}{R^{n}} \quad \forall n \in \mathbb{N}
$$

for any $R \in(0,1)$ and some constants $C_{2}, C_{3}>0$. The following result comes from [9, 13].

Lemma 3.3. Let $s \in(0,1)$ and let $\left(a_{n}\right)_{n \geq 0}$ be a sequence such that

$$
\left|a_{n}\right| \leq C \frac{n !^{s}}{R^{n}} \quad \forall n \geq 0
$$

for some constants $C, R>0$. Then the function $f(x)=\sum_{n \geq 0} a_{n} \frac{x^{n}}{n !}$ is Gevrey of order $s$ on $[-l, l]$ for all $l>0$, with

$$
\left|f^{(m)}(x)\right| \leq C\left(\sum_{k \geq 0} \frac{\left(2^{s} l\right)^{k}}{R^{k} k !^{1-s}}\right) \frac{2^{s m}}{R^{m}} m !^{s} \quad \forall m \in \mathbb{N}, \forall x \in[-l, l] .
$$

It follows from (3.13) and Lemma 3.3 that the Airy function is Gevrey of order $1 / 3$ on each interval $[-l, l]$ with

$$
\left|\mathrm{Ai}^{(m)}(x)\right| \leq C_{4}(R, l) \frac{2^{\frac{m}{3}}}{R^{m}} m !^{\frac{1}{3}} \quad \forall m \in \mathbb{N}, \forall x \in[-l, l] .
$$

Claim 4 is proved. Let us go back to the proof of Proposition 2.3. The fundamental solution of the simplified linear Korteweg-de Vries equation (2.31) is given by

$$
E(x, t)=\frac{1}{(3 t)^{\frac{1}{3}}} \mathrm{Ai}\left(\frac{x}{(3 t)^{\frac{1}{3}}}\right),
$$

so that the solution of (2.31)-(2.32) for an initial value $y_{0} \in L^{2}(\mathbb{R})$ supported in $[-L, L]$ reads

$$
y(x, t)=\left[E(., t) * y_{0}\right](x)=\frac{1}{(3 t)^{\frac{1}{3}}} \int_{-L}^{L} \operatorname{Ai}\left(\frac{x-s}{(3 t)^{\frac{1}{3}}}\right) y_{0}(s) d s .
$$

It is clear that $y$ is of class $C^{\infty}$ on $\mathbb{R}_{x} \times(0,+\infty)_{t}$. Pick any $l>0$ and any $0<\varepsilon<T$. Since $y$ solves (2.31), we have for all $x \in[-l, l], t \in[\varepsilon, T]$ and $p, q \in \mathbb{N}$ that

$$
\begin{aligned}
\left|\partial_{x}^{p} \partial_{t}^{q} y(x, t)\right| & =\left|\partial_{x}^{p+3 q} y(x, t)\right| \\
& \leq K \frac{2^{\frac{p+3 q}{3}}}{(3 t)^{\frac{1+p+3 q}{3}} R^{p+3 q}}(p+3 q) ! !^{\frac{1}{3}}\left\|y_{0}\right\|_{L^{1}(-L, L)} \\
& \leq K \frac{2^{\frac{p+3 q}{3}}}{(3 \varepsilon)^{\frac{1+p+3 q}{3}} R^{p+3 q}}\left(2^{p+3 q} p !(3 q) !\right)^{\frac{1}{3}}\left\|y_{0}\right\|_{L^{1}(-L, L)} \\
& \leq \frac{K^{\prime}}{R_{1}^{p} R_{2}^{q}} p^{\frac{1}{3}} q !\left\|y_{0}\right\|_{L^{1}(-L, L)}
\end{aligned}
$$


for some constants $K, K^{\prime}, R_{1}, R_{2}>0$ which depend on $l, L, \varepsilon$ and $T$. The proof of Proposition 2.3 is complete.

\section{ACKNOWLEDGEMENTS}

This work was done when the second author (IR) was visiting CAS, MINES ParisTech. The third author (LR) was supported by the ANR project Finite4SoS (ANR-15-CE23-0007).

\section{REFERENCES}

[1] M. A. Caicedo, R. de A. Capistrano Filho, B.-Y. Zhang, Neumann boundary controllability of the Korteweg-de Vries equation on a bounded domain, SIAM J. Control Optim. 55 (2017), no. 6, 3503-3532.

[2] E. Cerpa, Control of a Korteweg-de Vries equation: a tutorial, Math. Control Relat. Fields 4 (2014), no. 1, 45-99.

[3] E. Cerpa, I. Rivas, B.-Y. Zhang, Boundary controllability of the Korteweg-de Vries equation on a bounded domain, SIAM J. Control Optim. 51 (2013), no. 4, 2976-3010.

[4] J. Dardé, S. Ervedoza, On the reachable set for the one-dimensional heat equation, SIAM J. Control Optim., to appear.

[5] O. Glass, S. Guerrero, Some exact controllability results for the linear KdV equation and uniform controllability in the zero-dispersion limit, Asymptotic Analysis 60 (2008), 61-100.

[6] O. Glass, S. Guerrero, Controllability of the Korteweg-de Vries equation from the right Dirichlet boundary condition, System \& Control Letters 59 (2010) 390-395.

[7] L. Hörmander, The analysis of linear partial differential operators. I. Berlin Heidelberg New York, Springer 1983.

[8] P. Martin, L. Rosier, P. Rouchon, Null controllability of the heat equation using flatness, Automatica 50 (2014), No. 12, 3067-3076.

[9] P. Martin, L. Rosier, P. Rouchon, Controllability of the 1D Schrödinger equation by the flatness approach, IFAC Proceedings Volumes, 47(3): 646-651, 2014. 19th IFAC World Congress.

[10] P. Martin, L. Rosier, P. Rouchon, Null controllability of one-dimensional parabolic equations be the flatness approach, SIAM J. Control Optim. 54 (2016), No. 1, 198-220.

[11] P. Martin, L. Rosier, P. Rouchon, On the reachable states for the boundary control of the heat equation, Appl. Math. Res. Express. AMRX 2016, no. 2, 181-216.

[12] P. Martin, L. Rosier, P. Rouchon, Controllability of the 1D Schrödinger equation using flatness, Automatica 91 (2018), 208-216.

[13] P. Martin, L. Rosier, P. Rouchon, Controllability of some evolution equations by the flatness approach, in press.

[14] A. F. Pazoto, L. Rosier, Stabilization of a Boussinesq system of KdV-KdV type, Systems \& Control Letters 57 (2008), $595-601$.

[15] L. Rosier, Exact boundary controllability for the Korteweg-de Vries equation on a bounded domain, ESAIM Control Optim. Calc. Var. 2 (1997), 33-55.

[16] L. Rosier, A fundamental solution supported in a strip for a dispersive equation. Special issue in memory of Jacques-Louis Lions, Comput. Appl. Math. 21 (2002), no. 1, 355-367.

[17] L. Rosier, Control of the surface of a fluid by a wavemaker, ESAIM Control Optim. Calc. Var. 10 (3) (2004), 346-380.

[18] L. Rosier, B.-Y. Zhang, Control and stabilization of the Korteweg-de Vries equation: recent progresses. J. Syst. Sci. Complex. 22 (2009), no. 4, 647-682. 
Centre Automatique et Systèmes (CAS), Mines ParisTech, PSL Research University, 60 Boulevard SAINT-MiCHEL, 75272 PARIS CEDEX 06, FRANCE

E-mail address: philippe.martin@mines-paristech.fr

Universidad del Valle, Ciudadela Universitaria Meléndez, Calle 13 No. 100-00, A.A. 25360, Cali, COLOMBia

E-mail address: ivonne.rivasl@correounivalle.edu.co

Centre Automatique et Systèmes (CAS) and Centre de Robotique, Mines Paristech, PSL Research University, 60 Boulevard Saint-Michel, 75272 Paris Cedex 06, France

E-mail address: lionel.rosier@mines-paristech.fr

Centre Automatique et Systèmes (CAS), Mines Paristech, PSL Research University, 60 Boulevard SAINT-Michel, 75272 PARIS CEDEX 06, FranCE

E-mail address: pierre.rouchon@mines-paristech.fr 